Gazi University
Journal of Science
http://dergipark.gov.tr/gujs

\title{
A Suggestion System According to Fabric Control Time
}

\author{
Fatma Gunseli YASAR CIKLACANDIR ${ }^{1, *}$ (D) ${\text { Semih } \text { UTKU }^{2}}^{(1)}$ \\ ${ }^{I}$ Izmir Katip Celebi University, Computer Engineering, Izmir, Turkey \\ ${ }^{2}$ Dokuz Eylul University, Computer Engineering, Izmir, Turkey
}

\section{Highlights}

- This paper focuses on classification process of defects on fabrics.

- Fabric control time plays an important role in which methods to choose.

- The fabric production quality increases through the successes of the systems suggested.

\begin{tabular}{l} 
Article Info \\
Received: 01 Dec 2020 \\
Accepted: 02 Jan 2022 \\
Keywords \\
\hline Fabric defect \\
Feature extraction \\
Defect recognition
\end{tabular}

\section{INTRODUCTION}

As the importance of fashion increased, the importance of the textile industry also increased. In order to survive in the textile industry, it is necessary to produce high-quality fabrics that are suitable for the fashion of the time. Wefts and warps are regularly woven in a defect-free fabric [1]. Defects that may occur on fabrics for various reasons reduce the quality of the fabrics. In many factories, trained operators are employed to find defects on the fabrics and to prevent the causes of new defects to occur. The development of image processing has automated the process of detecting and identifying fabric defects. Much effort has been performed in recent years for this automation process.

Many studies in this area use artificial neural network (ANN) to perform defect classification. Kaynar et al. compare the performances of Local Binary Pattern (LBP) and Gray Level Co-occurrence Matrix (GLCM) [2]. GLCM has higher rates of accuracy, sensitivity, and specificity than LBP. Zhang et al. use the combination of LBP and GLCM for feature extraction [3]. The developed system has superiority in classification using backpropagation neural network (BPNN) compared with LBP only, GLCM only, geometrical characteristic, Fourier transform, and wavelet transformation. Huang and Chen combine neural network (NN) and fuzzy logic [4]. It is demonstrated that neural-fuzzy systems have higher performances than neural systems. With the emergence of deep learning, the number of studies based on it is increasing day by day [5-8] (Beljadid et al. 2020, Wang et al. 2020, Jing et al. 2020, Wei et al. 2019). Wei et al. emphasize that $\mathrm{CNN}$ applications are not yet common in this area and they propose a system that also works well for small sample sizes [8].

Support Vector Machines are another well-known classification method used in this area. Ghosh et al. have used SVM and they conclude that SVM classifies the defects with a higher accuracy rate compared to other

*Corresponding author, e-mail: fatmagunseli.yasar@ikc.edu.tr 
machine learning methods like artificial neural network (ANN) [9]. Salem and Abdelkrim use GLCM, LBP, and Local Phase Quantization (LPQ) as feature extraction methods [10]. They use SVM for the classification of the defects. LBP has been the best method in this study. Cuifang et al. use a pyramid histogram of edge orientation gradients (PHOG) for feature extraction [11]. Then, they use SVM like [910]. PHOG allows extracting the best performing features compared to the methods of HOG (Histogram of Oriented Gradients) and SIFT (Scale-Invariant Feature Transform). Abdellah et al. combine genetic algorithm (GA) and SVM [12]. Salem and Nasri compare the performances of LBP and GLCM [13]. SVM has been used as a classifier. LBP gives more effective results in terms of time and accuracy. Features have been extracted from the images using the methods of the histogram, co-occurrence matrix, and shape descriptor in the study of Murino et al. [14]. These features have been classified using SVM. It is seen that the classifiers such as K-Nearest Neighbor (KNN) and Random Forest (RF) are also used in this field. Yildiz and Buldu extract the features of the images using Principal Component Analysis (PCA) and classify the defects using KNN [15]. Patil and Patil combine the methods of Discrete Wavelet Transform (DWT) and KNN [16]. Deotale and Sarode use GLCM and Gabor Wavelet for feature extraction [17]. They use random decision forest (RDF) to classify the images as defective or non-defective.

In this paper, an automatic fabric defect classification system based on machine learning is proposed so as to solve the fabric defect detection problems. The performances of two different feature extraction methods and the performances of two different classification algorithms have been investigated on non-patterned fabrics. It has been tried to classify three different fabric defect types (broken end, broken yard, and fuzzyball). The paper is organized as follows. In Section 2, the methods of feature extraction and classification and the dataset which we have used have been mentioned. Section 3 mentions the proposed system. The experimental results are discussed in Section 4. Finally, Section 5 summarizes the concluding remarks.

\section{MATERIAL METHOD}

\subsection{Dataset}

The dataset consists of 23 images of three different fabric defects (broken end, broken yard, and fuzzyball). There are seven images for the defect of broken end, seven images for the defect of broken yard, nine images for the defect of fuzzyball. These images have been taken from the database of Silvestre-Blanes et al. [18]. They are $4096 \times 256$ pixels. Sample images after preprocessing operations like resizing are given in Figure 1. The dataset consists of 23 images of three different fabric defects (broken end, broken yard, and fuzzyball.

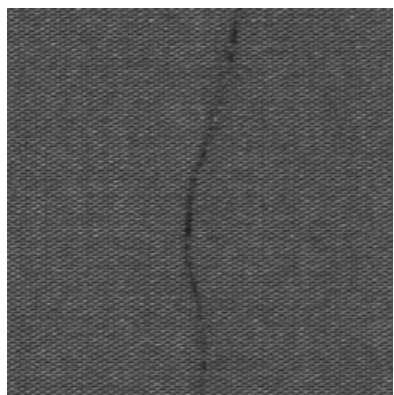

(a)

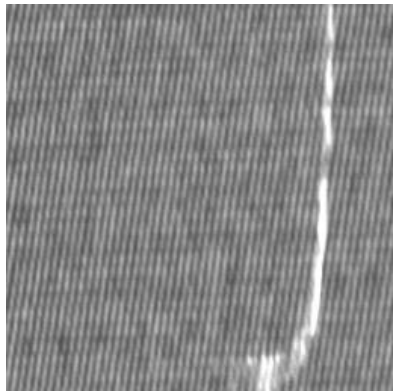

(b)

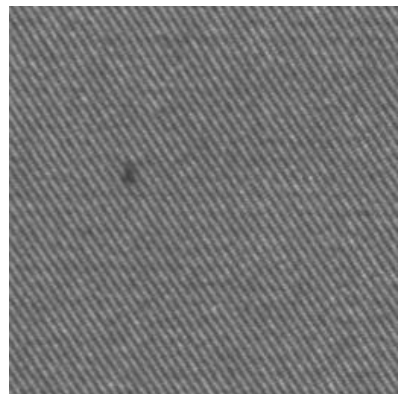

(c)

Figure 1. Fabric defects (a) Broken end (b) Broken yard (c) Fuzzyball

Up to three levels of subheadings can be used (2., 2.1., 2.1.1. etc). For further degrees of subheadings, do not use numbers, but simply give the heading in italics (Heading instead of 2.1.1.1. Heading). Single blank line must be left between titles, subtitles and the text body as in the paragraph separation. Italic font can be used for emphasis within the text. 


\subsection{Feature Extraction Methods}

\section{Principal Component Analysis (PCA)}

The PCA is a statistical technique widely used for data reduction, image compression, and feature extraction [19]. It is used in many fields. In this study, PCA has been used for feature extraction. It is based on the conversion of many number of variables to a small number of variables which are unrelated to each other. These variables obtained are called the principal components of the data.

The steps for applying the PCA to a dataset are as in Figure 2. Suppose, $X$ is a dataset. Average of all elements in the dataset $X$ is calculated. Then, the calculated average is subtracted from each element. Thus, a normalized data set $X^{\prime}$ is obtained. Covariance matrix $C$ is calculated using the elements in $X^{\prime}$ and their transposes. After calculating $\lambda$ and $\mathrm{V}$, a new data set is obtained.

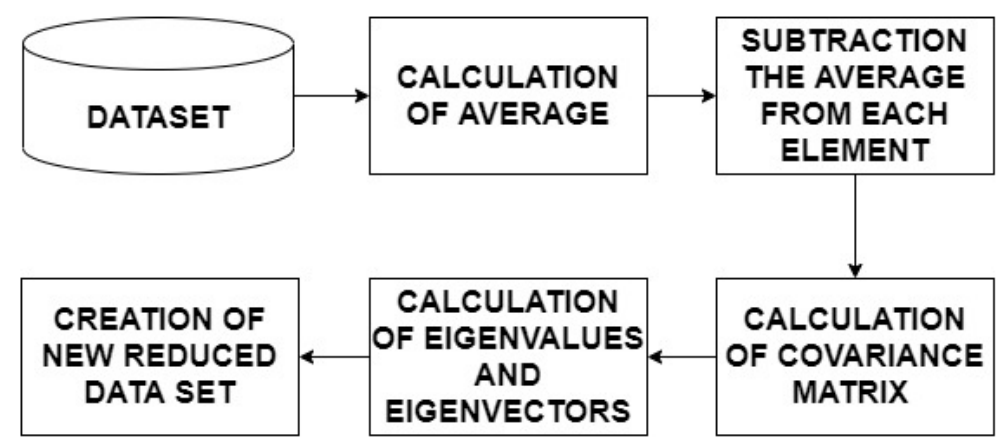

Figure 2. The flow chart of PCA

\section{Local Binary Pattern (LBP)}

The LBP is another well-known feature extraction method. The number of features extracted in this algorithm is determined according to the size of the selected window [2]. The LBP is based on sequential binary comparisons between the pixel values of center and neighbors of it [20]. Equation (1) is used to calculate LBP of images [21]. $x_{C}$ is center pixel, and $x_{i}$ is any neighbor of it. $\mathrm{R}$ is the distance of neighbors from the center pixel, and $\mathrm{P}$ is the number of neighbors of $x_{C}$. Each pixel of image is labeled by binarizing the difference between the center and its neighbors using Equation (2)

$$
\begin{aligned}
& \operatorname{LBP}_{\mathrm{P}, \mathrm{R}}\left(\mathrm{x}_{\mathrm{C}}\right)=\sum_{\mathrm{i}=0}^{\mathrm{P}-1} \mathrm{u}\left(\mathrm{x}_{\mathrm{i}}-\mathrm{x}_{\mathrm{C}}\right) 2^{\mathrm{i}}, \\
& \mathrm{u}(\mathrm{y})=\left\{\begin{array}{l}
1, \text { If } \mathrm{y} \geq 0 \\
0, \text { If } \mathrm{y}<0
\end{array}\right.
\end{aligned}
$$

\subsection{Classification Methods}

\section{K-Nearest Neighbor $(K N N)$}

The K-NN is one of the supervised learning algorithms. $\mathrm{K}$ is an input in the algorithm. A new element is assigned to the class according to the $\mathrm{K}$ closest neighbors in the data set. A distance metric is used to find closest neighbors. The metrics of Euclidean (Equation (3)), Minkowski (Equation (4)), and Manhattan (Equation (5)) are some of the most well-known distance metrics. $x=\left(x_{1}, x_{2}, \ldots, x_{n}\right)$ and $y=$ $\left(y_{1}, y_{2}, \ldots, y_{n}\right)$ are two elements in the data set. Euclidean distance metric has been used in this study.

$$
\begin{aligned}
& \mathrm{d}_{\text {Euclidean }}(\mathrm{x}, \mathrm{y})=\sqrt{\sum_{\mathrm{i}=1}^{\mathrm{n}}\left(\mathrm{x}_{\mathrm{i}}-\mathrm{y}_{\mathrm{i}}\right)^{2}} \\
& \mathrm{~d}_{\text {Minkowski }}(\mathrm{x}, \mathrm{y})=\left(\sum_{\mathrm{i}=1}^{\mathrm{n}}\left|\mathrm{x}_{\mathrm{i}}-\mathrm{y}_{\mathrm{i}}\right|^{\mathrm{p}}\right)^{1 / p}
\end{aligned}
$$


$\mathrm{d}_{\text {Manhattan }}(\mathrm{x}, \mathrm{y})=\sum_{\mathrm{i}=1}^{\mathrm{n}}\left|\mathrm{x}_{\mathrm{i}}-\mathrm{y}_{\mathrm{i}}\right|$

Support Vector Machine (SVM)

The SVM is another supervised learning algorithm. The purpose of SVM is to find the hyperplane that can optimally separates two classes from each other (Figure 3). The optimal hyperplane is the farthest plane to the nearest data points of the classes.

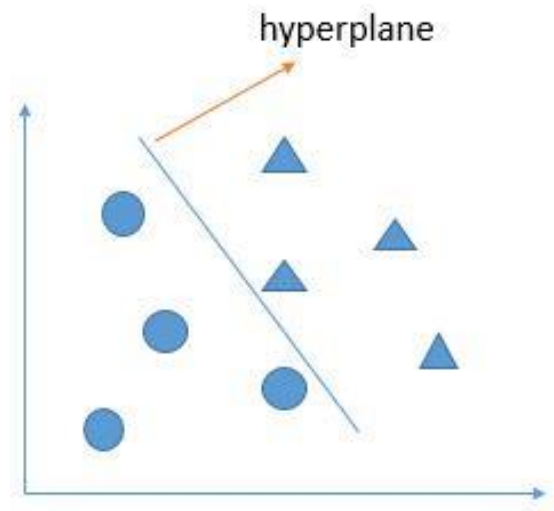

Figure 3. SVM

\section{THE PROPOSED METHOD}

As mentioned before, we aims fabric defect classification. For this purpose, more than one method has been compared and it has been tried to reach the algorithm that gives the optimal result. The flowchart of the system are given in Figure 4. Preprocessing operations consist of resizing and conversion from RGB to double. Then, the continuation of the study consists of two main phases: feature extraction phase and classification phase. The methods of PCA and LBP in feature extraction phase, and the methods of KNN and SVM in classification phase have been compared in terms of performance.

The performances of the methods have been evaluated using their accuracy values. Accuracy is the ratio of $\mathrm{TP}+\mathrm{TN}$ and $\mathrm{TP}+\mathrm{FN}+\mathrm{FP}+\mathrm{TN}$ (Equation (6)). TP, FN, FP, and TN are the numbers of True Positives, False Negatives, False Positives, and True Negatives. In Table 1, the instances in predicted classes represent the columns while the instances in actual classes represent the rows in the matrix [22]. Consider a system that detects whether there are any defects in the fabric. If there is a defect in the fabric, and the algorithm predicts a defect, the result of the test is TP [23]. Similarly, if there is not any defect, and the algorithm does not predict a defect, the result of the test is TN. If the algorithm predicts the presence of a defect which actually has no such defect, the test result is false positive (FP). Similarly, if the algorithm does not predict the presence of a defect in the fabric which actually has a defect, the test result is false positive (FN).

Accuracy $=\frac{\mathrm{TP}+\mathrm{TN}}{\mathrm{TP}+\mathrm{FN}+\mathrm{FP}+\mathrm{TN}}$ 


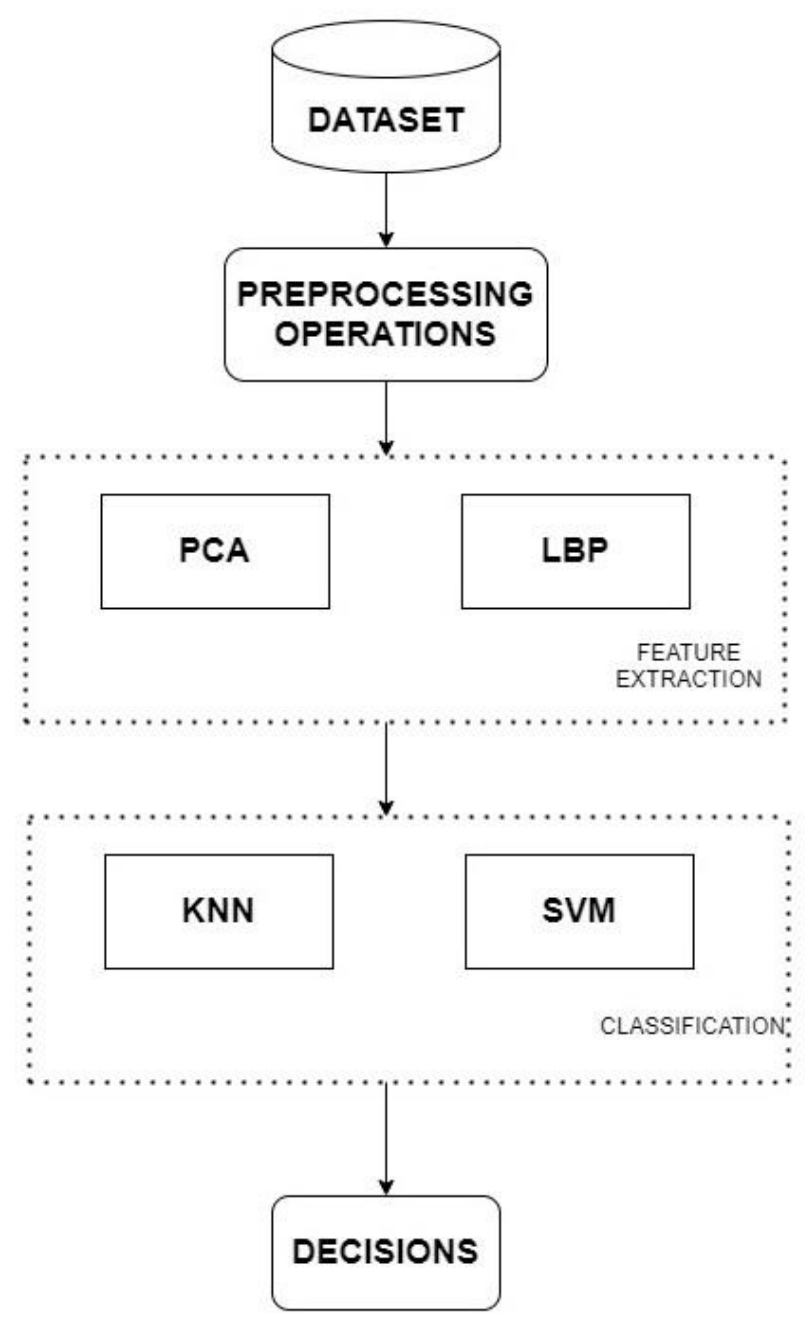

Figure 4. Flow chart of the system

Table 1. TP, FN, FP, and TN

\begin{tabular}{|c|c|c|c|}
\cline { 3 - 4 } \multicolumn{2}{c|}{} & \multicolumn{2}{c|}{ Predicted } \\
\cline { 3 - 4 } Actual & $\mathrm{P}$ & $\mathrm{P}$ & $\mathrm{N}$ \\
\cline { 2 - 4 } & $\mathrm{N}$ & $\mathrm{TP}$ & $\mathrm{FN}$ \\
\hline
\end{tabular}

\section{THE RESEARCH FINDINGS AND DISCUSSION}

The study was carried out on a personal computer with Intel(R) Core(TM) i7-6700HQ CPU. Coding of the program was done in Matlab 2019. 10-fold cross-validation (CV) has been used in order to compare the methods. Classification performances of the methods are given in Table 2.

It is seen that KNN and SVM, which perform equally when using LBP, perform differently when PCA is used. K-NN has correctly classified all images with the features extracted using PCA. When using LBP, the accuracy rate of KNN has decreased while the accuracy rate of SVM has increased. In addition to that, the average accuracy rate of KNN (97.8261\%) is higher than the average of SVM (91.3044\%). 
Table 2. Classification performances of the methods

\begin{tabular}{|l|c|c|c|c|}
\hline Method & Precision & Recall & F1-Measure & Accuracy \\
\hline PCA\& KNN & $100 \%$ & $100 \%$ & $100 \%$ & $100 \%$ \\
\hline LBP\& KNN & $95.6522 \%$ & $100 \%$ & $97.7778 \%$ & $95.6522 \%$ \\
\hline PCA\& SVM & $100 \%$ & $86.3636 \%$ & $92.6829 \%$ & $86.9565 \%$ \\
\hline LBP\& SVM & $95.6522 \%$ & $100 \%$ & $97.7778 \%$ & $95.6522 \%$ \\
\hline
\end{tabular}

The metric of time is of great importance in the system to be developed to detect defects during fabric production. If the automatic control will be carried out after production rather than during production, higher performance may be desired. Here, time may be less important and the differences in seconds in the running times of the algorithms may be negligible. For this purpose, the rates of performance and execution time have been examined and interpreted in Table 3. Since the number of features used by PCA is higher than LBP, its execution time is longer ( 0.974 seconds). When the execution times are compared in terms of classifiers, KNN results in a shorter time than SVM (0.611 seconds).

Finally, the accuracy/time rates of the methods have been investigated in order to compare them in terms of both accuracy and time. Accuracy is divided by time since the goal is maximum accuracy in the shortest time. Tables 2 and 3 should be evaluated together in order to find the most suitable system for the setup to be applied. According to the table, the rate of LBP\&KNN is the highest rate (2.0137). This is because the execution time of LBP is low. In this case, the method with high results in a short time is LBP\&KNN. This combination will be more effective in mechanisms that perform control during production, where time is as valuable as accuracy. Accuracy is the first priority metric compared to time in the mechanisms that control after production, not during production. In these mechanisms, time is more flexible. Therefore, it will be best to use PCA\& KNN, which has approximately twice the execution time of LBP\& KNN. PCA\& KNN's high execution time does not change the fact that it has $100 \%$ accuracy rate.

Table 3. Time \& Accuracy/Time

\begin{tabular}{|l|c|c|}
\hline Method & Execution Times $(\mathrm{sec})$ & Accuracy/Time \\
\hline PCA\& KNN & 0.747 seconds & 1.3386 \\
\hline LBP\& KNN & 0.475 seconds & 2.0137 \\
\hline PCA\& SVM & 1.201 seconds & 0.7240 \\
\hline LBP\& SVM & 0.959 seconds & 0.9974 \\
\hline
\end{tabular}

Detailed information and results of some similar studies are given in Table 4. The lowest accuracy rates were obtained in [13].

Table 4. Comparison with other studies

\begin{tabular}{|c|c|c|c|}
\hline Study & Dataset & Methods & Accuracy \\
\hline [2] & Tilda & $\begin{array}{l}\mathrm{LBP}+\mathrm{NN} \\
\mathrm{GLCM}+\mathrm{NN}\end{array}$ & $\begin{array}{l}90.6 \% \\
91.1 \%\end{array}$ \\
\hline [3] & Tilda & LBP+GLCM+BPNN & $97.6 \%$ \\
\hline [8] & Their dataset & $\begin{array}{l}\text { KNN } \\
\text { MLP } \\
\text { SVM } \\
\text { CNN } \\
\text { CS-CNN }\end{array}$ & $\begin{array}{l}96.0 \% \\
89.4 \% \\
91.7 \% \\
89.7 \% \\
97.9 \% \\
\end{array}$ \\
\hline [10] & Tilda & $\begin{array}{l}\text { GLCM+SVM } \\
\text { LBP+SVM } \\
\text { LPQ+SVM }\end{array}$ & $\begin{array}{l}98.25 \% \\
97.25 \% \\
88.5 \% \\
\end{array}$ \\
\hline [11] & Tilda & $\begin{array}{l}\text { SIFT+SVM } \\
\text { HOG+SVM } \\
\text { PHOG+SVM }\end{array}$ & $\begin{array}{l}72.59 \% \\
90.61 \% \\
92.61 \% \\
\end{array}$ \\
\hline [12] & Their dataset & $\mathrm{GA}+\mathrm{SVM}$ & $96.15 \%$ \\
\hline
\end{tabular}




\begin{tabular}{|c|l|l|c|}
\hline$[13]$ & Tilda & $\begin{array}{l}\text { GLCM+SVM } \\
\text { LBP+SVM } \\
\text { GLCM+ LBP+SVM }\end{array}$ & $\begin{array}{r}77.5 \% \\
82.93 \% \\
86.70 \%\end{array}$ \\
\hline \multirow{3}{*}[14]{} & Parvis, Tilda & $\begin{array}{l}\text { Histogram, shape descriptors } \\
\text { and the cooccurrence } \\
\text { matrices+SVM }\end{array}$ & $\begin{array}{r}99.11 \% \text { (for Parvis) } \\
92.87 \% \text { (for Tilda) }\end{array}$ \\
\hline$[15]$ & Their dataset & GLCM+KNN & $95 \%$ \\
\hline$[16]$ & Their dataset & DWT+KNN & $95 \%$ \\
\hline \multirow{2}{*}[17]{} & A dataset taken & GLCM+RDF & $98.15 \%$ \\
\hline & from [24] & PCA+KNN & $100 \%$ \\
& A dataset taken & PCA+SVM & $86.96 \%$ \\
& from [18] & LBP+KNN & $95.65 \%$ \\
& & LBP+SVM & $95.65 \%$ \\
\hline
\end{tabular}

\section{RESULTS}

If the goal is an automatic system development, the completion time of the algorithm can be as valuable as the performance. Especially in online systems, performance measurement should not be the only evaluation criteria. However, this situation has been neglected in most studies in the literature and they have focused on performance only.

Some of the fabric factories that do automatic control carry out defect control after production, while others do defect control during production. We do not think it is correct to suggest a single system for both types of factories. Therefore, there are suggestion systems for them in this study. It may be more effective to use a system based on the time of the control. For this purpose, the performance/time values of different methods have been also compared besides classical performance metrics such as precision, recall, f1measure, accuracy. The performances of two different feature extraction methods (PCA, and LBP) and two different classification methods (KNN, and SVM) have been investigated in the paper. 10-fold crossvalidation has been used as performance measurement. It has not been found in the studies in the literature that the rate of accuracy/time has been examined. In this study, six different parameters have been investigated (precision, recall, f1-measure, accuracy, time, and accuracy/time). High accuracy, low time, and high accuracy/time rate are required in the systems designed.

According to the tests, the combination of PCA and KNN achieves 100\% accuracy. This combination results in 0.747 seconds. The accuracy/time rate is 1.3386 . The combination of LBP and KNN achieves $95.6522 \%$ accuracy. This combination results in 0.475 seconds. The accuracy/time rate is 2.0137 . The combination of PCA and SVM achieves $86.9565 \%$ accuracy. This combination results in 1.201 seconds. Accuracy/time rate is 0.7240 . The combination of LBP and SVM achieves $95.6522 \%$ accuracy. This combination results in 0.959 seconds. Accuracy/time rate is 0.9974 . Consequently, it can be said that the combination of LBP and KNN is suggested if the control will be made during fabric production, while the combination of PCA and $\mathrm{KNN}$ is recommended if it will be made after fabric production. Because LBP responds in a shorter time compared to PCA. However, the 100\% accuracy rate achieved in the combination of PCA and KNN cannot be ignored.

The fabric production quality increases through the successes of the systems suggested. In addition, while meeting customer satisfaction, productivity will increase and profit loss due to defective fabrics will be minimized.

In future studies, we will compare the methods used in this study with deep learning-based methods by using a larger data set. The size of the data set limits the methods to be used in the studies carried out to find fabric defects. If there are many elements belonging to each class, deep learning methods can be used. Therefore, in our future work, we first aim to reach a large data set or to create our own dataset. Then, we aim to improve the disadvantages of deep learning-based methods such as responding in a long time and 
not being able to find more than one defect at the same time. Because, as emphasized in this study, time is also important in studies to find fabric defects. To sum up, the summary of the developed study is given in Table 5.

Table 5. Summary Table

\begin{abstract}
What do we already know about this topic?
There are some studies [2-17] about the automatic systems for fabric defect classification. Some of the fabric factories that do automatic control carry out after production, while others do defect control during production. However, there is no system that can classify the defects according to the time of defect control.
\end{abstract}

\title{
How does your research contribute to the field?
}

How accurate is it to use the same system in mechanisms that perform control at different times? In this paper, a customized system is suggested. The system will have the following advantages:

The combination of LBP and K-NN is suggested if the control will be made during fabric production.

The combination of PCA and K-NN is recommended if it will be made after fabric production.

While only performance criterion determines success in previous studies, execution time and performance/time are also considered in this study.

\section{What are your research's implications toward theory, practice?}

The success of the KNN algorithm cannot be ignored when using PCA. In addition, both KNN and SVM have the same success when LBP is used. Time and performance/time criteria have been distinctive here. It is recommended to consider all three criteria (performance, time, and performance/time) in the systems to be developed. The priority of performance criterion and time criterion may change according to the control time. Therefore, the correct combinations should be chosen, taking into account the control time.

\section{CONFLICTS OF INTEREST}

No conflict of interest was declared by the authors.

\section{REFERENCES}

[1] Shi, M., Jiang, S., Wang, H., Xu, B., "A simplified pulse-coupled neural network for adaptive segmentation of fabric defects", Machine Vision and Applications, 20(2): 131-138, (2009).

[2] Kaynar, O., Işik, Y. E., Görmez, Y., Demirkoparan, F., "Fabric defect detection with LBPGLCM", International Artificial Intelligence and Data Processing Symposium, 1-5, (2017).

[3] Zhang, L., Jing, J., Zhang, H., "Fabric defect classification based on LBP and GLCM", Journal of Fiber Bioengineering and Informatics, 8(1): 81-89, (2015).

[4] Huang, C. C., Chen, I. C., "Neural-fuzzy classification for fabric defects", Textile Research Journal, 71(3): 220-224, (2001).

[5] Beljadid, A., Tannouche, A., Balouki, A., "Application of deep learning for the detection of default in fabric texture", 6th International Conference on Optimization and Applications, Morocco, (2020).

[6] Wang, C., Wang, D., Wang, R., Leng, J., "Textile defect detection and classification based on deep convolution neural network", Developments of Artificial Intelligence Technologies in Computation and Robotics: Proceedings of the 14th International FLINS Conference, 1094-1101, (2020). 
[7] Jing, J., Wang, Z., Rätsch, M., Zhang, H., "Mobile-Unet: An efficient convolutional neural network for fabric defect detection", Textile Research Journal, 1-13, (2020).

[8] Wei, B., Hao, K., Tang, X. S., Ding, Y., "A new method using the convolutional neural network with compressive sensing for fabric defect classification based on small sample sizes", Textile Research Journal, 89(17): 3539-3555, (2019).

[9] Ghosh, A., Guha, T., Bhar, R. B., Das, S., "Pattern classification of fabric defects using support vector machines", International Journal of Clothing Science and Technology, 142-151, (2011).

[10] Salem, Y. B., Abdelkrim, M. N., "Texture classification of fabric defects using machine learning", International Journal of Electrical and Computer Engineering, 10(4): 4390, (2020).

[11] Cuifang, Z., Yu, C., Jiacheng, M., "Fabric defect detection algorithm based on PHOG and SVM", Indian Journal of Fibre \& Textile Research, 45(1): 123-126, (2020).

[12] Abdellah, H., Ahmed, R., Slimane, O., "Defect detection and identification in textile fabric by SVM method", International Organization of Scientific Research Journal of Engineering, 4(12): 69-77, (2014).

[13] Ben Salem, Y., Nasri, S., "Woven fabric defects detection based on texture classification algorithm", 8th International Multi-Conference on Systems, Signals \& Devices, Tunisia, (2011).

[14] Murino, V., Bicego, M., Rossi, I. A., "Statistical classification of raw textile defects", Proceedings of the 17th International Conference on Pattern Recognition, United States, (2004).

[15] Yildiz, K., Buldu, A., "Wavelet transform and principal component analysis in fabric defect detection and classification", Pamukkale University Journal of Engineering Science, 23: 622-627, (2017).

[16] Patil, M., Patil, S. R., "Fabric defect detection using discrete wavelet transform", International Research Journal of Engineering and Technology, 6(6): 3495-3499, (2017).

[17] Deotale, N. T., Sarode, T. K., "Fabric defect detection adopting combined GLCM, Gabor wavelet features and random decision forest", 3D Research, 10(1): 5, (2019).

[18] Silvestre-Blanes, J., Albero-Albero, T., Miralles, I., Pérez-Llorens, R., Moreno, J., "A Public Fabric Database for Defect Detection Methods and Results”, Autex Research Journal, 19(4): 363374, (2019).

[19] Yang, T. N., Wang, S. D., "Robust algorithms for principal component analysis", Pattern Recognition Letters, 20(9): 927-933, (1999).

[20] Tekeli, E., Cetin, M., Ercil, A., "A local binary patterns and shape priors based texture segmentation method", IEEE 15th Signal Processing and Communications Applications, Turkey, (2007).

[21] Nabiyev, V. V., Günay, A., "LBP yardımıyla görüntüdeki kişinin yaşının bulunması”, Çankaya University Journal of Science and Engineering, 8(1): 27-41, (2010).

[22] Horak, K., Klecka, J., Bostik, O., Davidek, D., "Classification of SURF image features by selected machine learning algorithms", 40th International Conference on Telecommunications and Signal Processing, Spain, (2017). 
[23] Zhu, W., Zeng, N., Wang, N., "Sensitivity, specificity, accuracy, associated confidence interval and ROC analysis with practical SAS implementations", NESUG proceedings: health care and life sciences, Baltimore, Maryland, 19: 67, (2010).

[24] Tabassian, M., Ghaderi, R., Ebrahimpour, R., "Knitted fabric defect classification for uncertain labels based on Dempster-Shafer theory of evidence", Expert Systems with Applications, 38(5), 5259-5267, (2011). 